\title{
QCD Analysis of the Polarized Deep-Inelastic World Data ${ }^{1}$
}

\author{
Johannes Blümlein and Helmut Böttcher \\ Deutsches Elektronen-Synchrotron, DESY, Platanenallee 6, D-15738 Zeuthen, Germany
}

\begin{abstract}
The results of a recent next-to-leading order QCD analysis of the world data on polarized deep inelastic scattering are reported. New parameterizations are derived for the quark and gluon distributions, accounting for the massive Wilson coefficient for the charm quarks, and the value of $\alpha_{s}\left(M_{Z}^{2}\right)$ is determined with correlated errors. We obtain $\alpha_{s}^{\mathrm{NLO}}\left(M_{Z}^{2}\right)=0.1132 \quad{ }_{-0.0095}^{+0.0056}$. Limits on potential higher twist contributions to the structure function $g_{1}\left(x, Q^{2}\right)$ are derived. We also compare to the results obtained by other groups.
\end{abstract}

Keywords: Polarized twist-2 parton distributions, strong coupling constant, higher twist terms PACS: 11.10.Hi,12.38.Qk,13.88.+e,14.65.Dw

\section{INTRODUCTION}

The composition of the nucleon's spin and the short distance behaviour of the partons inside strongly polarized nucleons in general constitutes one of the central research topics in QCD, with emphasis on the twist-2 contributions. They are explored both with perturbative and non-perturbative methods. During the last years the polarized deepinelastic scattering data have further improved [1-15]. In this note we report on a new next-to-leading order (NLO) QCD analysis of these data [16], updating earlier investigations [17]. At large enough four-momentum transfer $Q^{2}=-q^{2}$, the structure function $g_{1}\left(x, Q^{2}\right)$ mainly receives twist -2 contributions ${ }^{2}$ and is related to the polarized twist-2 parton distribution functions (PDFs). We analyze the structure function $g_{1}\left(x, Q^{2}\right)$, which is derived from the longitudinal polarization asymmetry accounting for a data-based description of the denominator function [19] and corresponding parameterizations for the longitudinal structure function, cf. [16]. In the present analysis we include the $O\left(\alpha_{s}\right)$ contribution due to charm quarks, $[20,21]^{3}$. The structure function $g_{2}\left(x, Q^{2}\right)$ is described at leading twist by the Wandzura-Wilczek relation $[23,21]$. The parameters of the polarized parton densities, which can be measured using the above data sets, are mandatorily determined with correlated errors along with the QCD scale $\Lambda_{\mathrm{QCD}}^{N_{f}=4}$. We also analyze potential contributions of higher twist and derive corresponding limits. Finally, a phenomenological parameterization of the polarized NLO PDFs is provided in terms of grids for the central values and the correlated errors, [24].

\footnotetext{
1 DESY 10-244, SFB/CPP-10-133. Supported in part by DFG SFB-TR-9.

2 Twist-3 contributions are connected by target mass effects, cf. [18].

3 The $O\left(\alpha_{s}^{2}\right)$ heavy flavor corrections are only known in the asymptotic region $Q^{2} \gg m^{2}$, [22].
} 


\section{THE ANALYSIS}

The NLO QCD analysis of the structure function $g_{1}\left(x, Q^{2}\right)$ is performed in Mellin space following the standard formalism, cf. e.g. [25], including the heavy quark corrections [26]. ${ }^{4}$ In this representation the evolution equations can be solved analytically, in both a fast and numerically precise way. Only one numerical integral around the singularities of the solution in the complex plane, located at the real axis left to an upper bound, has to be performed to represent $g_{1}\left(x, Q^{2}\right)$. As the data are located at low values of $Q^{2}$ target mass corrections are applied, cf. [18, 28]. For the deuteron targets a wave function correction is performed [29]. The parton distributions at the starting scale $Q_{0}^{2}=4 \mathrm{GeV}^{2}$ are parameterized by

$$
x \Delta f_{i}\left(x, Q_{0}^{2}\right)=\eta_{i} A_{i} x^{a_{i}}(1-x)^{b_{i}}\left(1+\gamma_{i} x\right)
$$

with $\eta_{i}$ the first moments. The present analysis parameterizes the sea quarks assuming approximate flavor $S U(3)$ symmetry. The deep-inelastic data alone cannot resolve the flavor dependence of the sea. Taking into account semi-inclusive data [30], and later on polarized Drell-Yan and di-muon data, will allow the determination of polarized sea quark distributions, similar to the unpolarized case. ${ }^{5} \eta_{u_{v}}$ and $\eta_{d_{v}}$ are fixed due to the neutron and hyperon- $\beta$ decay parameters $F$ and $D$, which are very well measured :

$$
\begin{array}{rll}
\eta_{u_{v}}-\eta_{d_{v}}=F+D & \text { and } & \eta_{u_{v}}+\eta_{d_{v}}=3 F+D \\
\eta_{u_{v}}=0.928 \pm 0.014 & \text { and } & \eta_{d_{v}}=-0.342 \pm 0.018
\end{array}
$$

The parameters in (1) cannot all be measured using the present data since for some the $\chi^{2}$-fit yields errors larger than $100 \%$. In case of the sea-quark and gluon density $\gamma_{i}$ is found to be compatible with zero. Furthermore the $a_{i}$-parameters of the distributions $\Delta q_{s}$ and $\Delta G$ are related by about $a_{\Delta G}=a_{\Delta q_{s}}+1$, which we use. The parameters $\gamma_{u_{v}}$ and $\gamma_{u_{v}}$ are fitted in an intial run and are then kept fixed as model parameters. For the large- $x$ parameters $b_{\Delta q_{s}}$ and $b_{\Delta G}$ we used the relation $b_{\Delta q_{s}} / b_{\Delta G}(\mathrm{pol})=b_{\Delta q_{s}} / b_{\Delta G}($ unpol $)=1.44$ and determine $b_{\Delta G}=5.61$ and $b_{\Delta q_{s}}=8.08$ in the fit. In the final fit 8 parameters are determined including $\Lambda_{\mathrm{QCD}}^{N_{f}=4}$. In Figure 1 we show the four distributions $\Delta u_{v}, \Delta d_{v}, \Delta q_{s}$ and $\Delta G$ at the input scale and compare them to other determinations. In Ref. [16] we provide the correlated errors. Due to this one may perform Gaussian error propagation for all observales based on polarized parton denstities predicting the PDF errors of these quantites. There we also compute a series of moments for the different parton densities, see Ref. [16], which can be compared to upcoming lattice simulations.

The nucleon spin is given by the relation

$$
\frac{1}{2}=\frac{1}{2}\langle\Delta \Sigma(x)\rangle_{0}+\langle\Delta G(x)\rangle_{0}+L_{q}+L_{g}
$$

\footnotetext{
${ }^{4}$ We refrain from carrying out small- $x$ resummations, since yet unknown subleading terms are very likely to cancel the leading order effects, cf. [27].

${ }^{5}$ For first analyses accounting for the flavor dependence of the sea quarks see [31].
} 

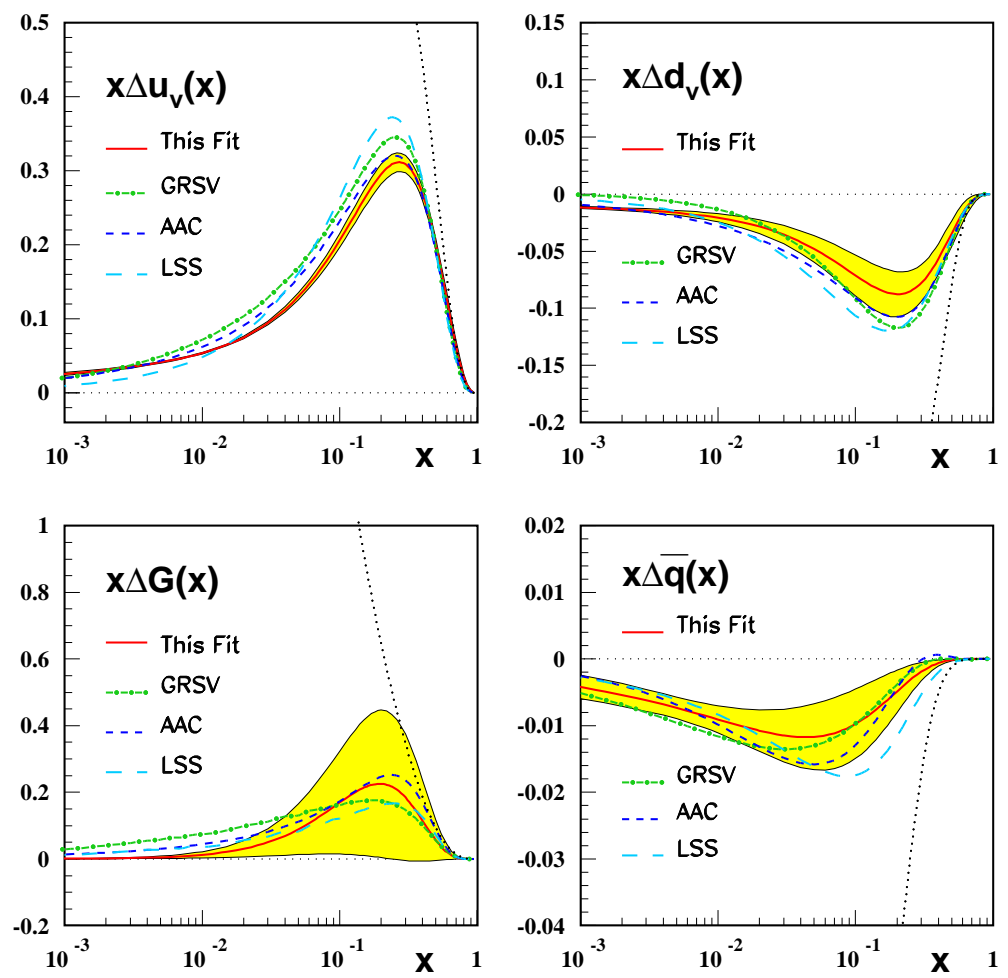

FIGURE 1. NLO polarized parton distributions at the input scale $Q_{0}^{2}=4.0 \mathrm{GeV}^{2}$ (solid line) compared to results obtained by GRSV (dashed-dotted line) [32], AAC (dashed line) [33], and LSS (long dashed line) [34]. The shaded areas represent the fully correlated $1 \sigma$ error bands calculated by Gaussian error propagation. The dotted line indicates the positivity bound using the parameterization [35]; from Ref. [16].

to the first moments of the polarized flavor singlet and gluon distributions and the quark and gluon angular momenta $L_{q, g}$. In the present analysis we obtain

$$
\begin{aligned}
\langle\Delta \Sigma(x)\rangle_{0} & =0.216 \pm 0.079 \\
\langle\Delta G(x)\rangle_{0} & =0.462 \pm 0.430
\end{aligned}
$$

which saturates the required value even for vanishing values for $L_{q}$ and $L_{g}$. However, the error on the gluon density is still rather large. Using the grids [24] one may perform predictions for polarized observables at hadron colliders which depend on the twist-2 parton distributions for RHIC or other machines planned for the future, as e.g. for the Drell-Yan process or pseudoscalar Higgs boson production [36] ${ }^{6}$.

In deep-inelastic QCD analyses it is important to determine the PDF-parameters at the initial scale $Q_{0}^{2}$ together with the QCD scale $\Lambda_{\mathrm{QCD}}$ since there are strong correlations, e.g. between the gluon-normalization and $\alpha_{S}\left(M_{Z}^{2}\right)$, but also to other parameters. We ob-

\footnotetext{
${ }^{6}$ For recent predictions in case of unpolarized $p(\bar{p}) p$-scattering at NNLO see [37].
} 


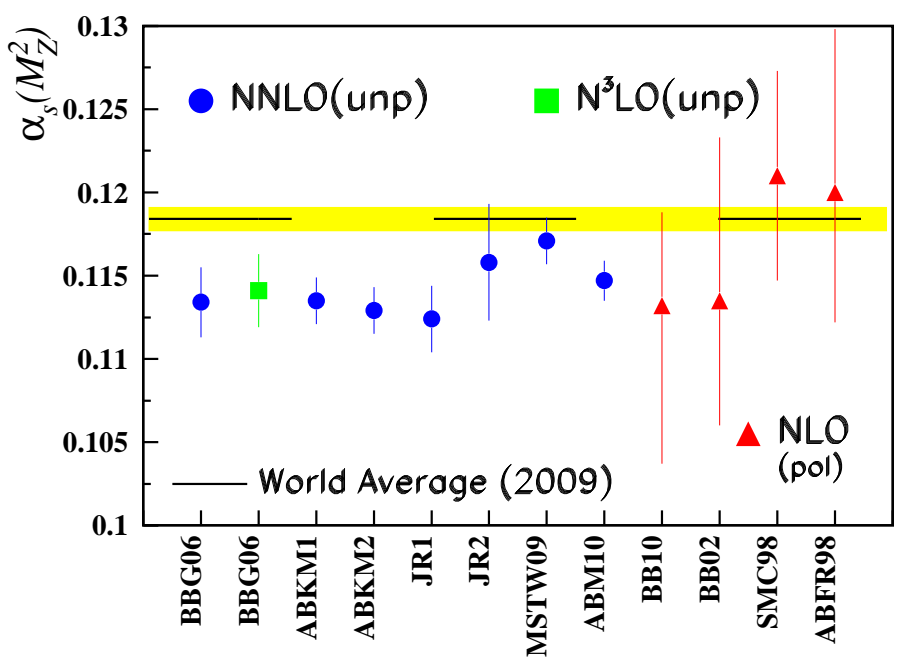

FIGURE 2. A summary of the current measurements of $\alpha_{s}\left(M_{Z}^{2}\right)$ from unpolarized and polarized DIS data, cf. Ref. [16] for details. Due to the size of errors we include only the results of NNLO and $\mathrm{N}^{3} \mathrm{LO}$ analyses in the unpolarized case, while those in the polarized case stem from NLO analyses. The yellow band marks the weighted world average of 2009, to which, however, measurements of $\alpha_{s}\left(M_{Z}^{2}\right)$ at NLO, NNLO and $\mathrm{N}^{3} \mathrm{LO}$ contribute [38].

tain

$$
\Lambda_{\mathrm{QCD}}^{(4)}=243 \pm 62(\exp ) \begin{array}{r}
-37 \\
+21
\end{array}(\mathrm{FS}) \begin{array}{r}
+46 \\
-87
\end{array}(\mathrm{RS}) \mathrm{MeV}
$$

The renormalization (RS) and factorization scales (FS) were varied by a factor of 2 around $Q^{2}$. Here we excluded values $\mu_{f, r}^{2}<1 \mathrm{GeV}^{2}$, unlike in Ref. [17], since at scales lower than $1 \mathrm{GeV}^{2}$ the perturbative description cannot be considered reliable anymore.

Correspondingly, one obtains

$$
\alpha_{s}\left(M_{Z}^{2}\right)=0.1132 \begin{aligned}
& +0.0043 \\
& -0.0051
\end{aligned}(\exp ) \begin{aligned}
& -0.0029 \\
& +0.0015
\end{aligned}(\mathrm{FS}) \begin{aligned}
& +0.0032 \\
& -0.0075
\end{aligned} \quad(\mathrm{RS})
$$

The errors are much larger than in the unpolarized case, at NNLO, where an accuracy of $O(1 \%)$ is reached. Still the central value is lower than the current world average and well comparable to the unpolarized values. In Figure 2 we summarize the current status of $\alpha_{s}\left(M_{Z}^{2}\right)$ measurements in deep-inelastic scattering. We would like to mention the results of the unpolarized NS-analysis [39] at $\mathrm{N}^{3} \mathrm{LO}:^{7}$

$$
\alpha_{s}\left(M_{Z}^{2}\right)=0.1141+0.0020
$$

and recent combined NS and singlet NNLO analyses [40, 41]

$$
\begin{aligned}
& \alpha_{s}\left(M_{Z}^{2}\right)=0.1124 \pm 0.0020 \\
& \alpha_{s}\left(M_{Z}^{2}\right)=0.1135 \pm 0.0014 .
\end{aligned}
$$

\footnotetext{
7 The corresponding NLO and NNLO values are $\alpha_{s}\left(M_{Z}^{2}\right)=0.1148 \pm 0.0019$ and $0.1134 \pm 0.0020$, respectively, pointing to the fact that the NNLO values are systematically smaller than the NLO values. Therefore it is problematic to average $\alpha_{s}$ values extracted at different orders.
} 


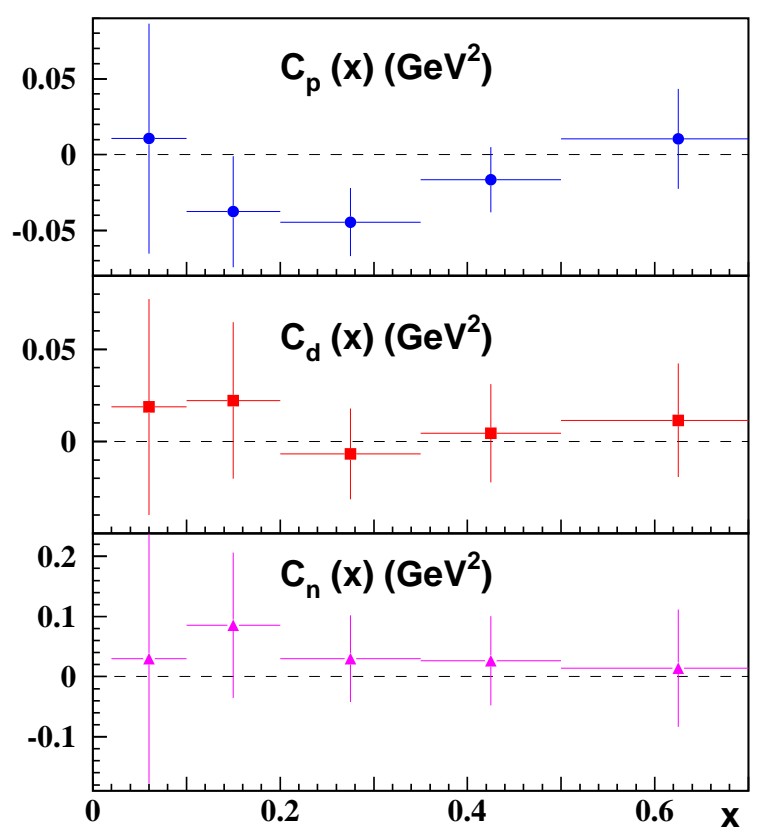

FIGURE 3. The additive higher twist coefficients $C_{p}(x), C_{d}(x)$ and $C_{n}(x)$ as a function of $x$.

Very recently, due to the inclusion of the combined H1+ZEUS data the latter value receives a slight change to

$$
\alpha_{S}\left(M_{Z}^{2}\right)=0.1147 \pm 0.0012
$$

reaching now the accuracy of $1 \%$, [42].

We also fitted additive higher twist terms to $g_{1}\left(x, Q^{2}\right)$, allowing for a model term $+C(x) /\left(Q^{2} / \mathrm{GeV}^{2}\right)$ besides the twist-2 contributions, to explore the corresponding structures in the region $x \leq 0.6$ for the proton- and deuteron targets, cf. Figure 3 . While in case of the deuteron target the result is fully compatible with zero, an effect of up to $2 \sigma$ is observed for one out of five bins in case for the proton target. This result is indicative mainly, since a measurement of the higher twist contributions would require a clear separation of the leading twist terms. This is possible in the unpolarized case, as has been shown in Refs. [43, 44]. This also requires even higher order corrections for the leading twist contributions. A comparable analysis in the polarized case has to be based on much more precise data in a far wider range of $Q^{2}$ which can be obtained at future colliders such as the EIC.

\section{REFERENCES}

1. J. Ashman et al. [EMC], Phys. Lett. B 206 (1988) 364; Nucl. Phys. B 328 (1989) 1.

2. P. L. Anthony et al. [E142 Collaboration], Phys. Rev. D 54 (1996) 6620 [arXiv:hep-ex/9610007].

3. K. Ackerstaff et al. [HERMES Collaboration], Phys. Lett. B 404 (1997) 383 [arXiv:hep-ex/9703005].

4. K. Abe et al. [E154 Collaboration], Phys. Rev. Lett. 79 (1997) 26 [arXiv:hep-ex/9705012].

5. B. Adeva et al. [Spin Muon Collaboration], Phys. Rev. D 58 (1998) 112001.

6. K. Abe et al. [E143 collaboration], Phys. Rev. D 58 (1998) 112003 [arXiv:hep-ph/9802357].

7. A. Airapetian et al. [HERMES Collaboration], Phys. Rev. D 75 (2007) 012007 [arXiv:hepex/0609039].

8. P. L. Anthony et al. [E155 Collaboration], Phys. Lett. B 463 (1999) 339 [arXiv:hep-ex/9904002].

9. P. L. Anthony et al. [E155 Collaboration], Phys. Lett. B 493 (2000) 19 [arXiv:hep-ph/0007248]. 
10. X. Zheng et al., The JLAB Hall A collaboration, Phys. Rev. C70 (2004) 065207.

11. V. Y. Alexakhin et al. [COMPASS Collaboration], Phys. Lett. B 647 (2007) 8 [arXiv:hepex/0609038].

12. K. V. Dharmawardane et al. [CLAS Collaboration], Phys. Lett. B 641 (2006) 11 [arXiv:nuclex/0605028].

13. The CLAS collaboration, private communication.

14. M. G. Alekseev et al. [The COMPASS Collaboration], arXiv:1001.4654 [hep-ex].

15. K. Abe et al. [E154 Collaboration], Phys. Lett. B 405 (1997) 180 [arXiv:hep-ph/9705344].

16. J. Blümlein and H. Böttcher, Nucl. Phys. B 841 (2010) 205 [arXiv:1005.3113 [hep-ph]].

17. J. Blümlein and H. Böttcher, Nucl. Phys. B 636 (2002) 225 [arXiv:hep-ph/0203155].

18. J. Blümlein and A. Tkabladze, Nucl. Phys. B 553 (1999) 427 [arXiv:hep-ph/9812478].

19. M. Arneodo et al. (NMC), Phys. Lett. B364 (1995) 107.

20. A. D. Watson, Z. Phys. C12 (1982) 123; W. Vogelsang, Z. Phys. C50 (1991) 275.

21. J. Blümlein, V. Ravindran and W. L. van Neerven, Phys. Rev. D 68 (2003) 114004 [arXiv:hep$\mathrm{ph} / 0304292]$.

22. M. Buza, Y. Matiounine, J. Smith et al., Nucl. Phys. B485 (1997) 420; [hep-ph/9608342]; I. Bierenbaum, J. Blümlein, S. Klein, [arXiv:0706.2738 [hep-ph]] and in preparation.

23. S. Wandzura, F. Wilczek, Phys. Lett. B72 (1977) 195.

24. http://www-zeuthen.desy.de/ blumlein

25. J. Blümlein and A. Vogt, Phys. Rev. D 58 (1998) 014020 [arXiv:hep-ph/9712546].

26. S. I. Alekhin, J. Blümlein, Phys. Lett. B594 (2004) 299. [hep-ph/0404034].

27. J. Blümlein and A. Vogt, Phys. Lett. B 370 (1996) 149 [arXiv:hep-ph/9510410]; Phys. Lett. B 386 (1996) 350 [arXiv:hep-ph/9606254].

28. J. Blümlein and A. Tkabladze, Nucl. Phys. Proc. Suppl. 79 (1999) 541 [arXiv:hep-ph/9905524].

29. M. Lacombe, B. Loiseau, R. Vinh Mau, J. Cote, P. Pires and R. de Tourreil, Phys. Lett. B 101 (1981) 139; W. W. Buck and F. Gross, Phys. Rev. D 20 (1979) 2361; M. J. Zuilhof and J. A. Tjon, Phys. Rev. C 22 (1980) 2369; R. Machleidt, K. Holinde and C. Elster, Phys. Rept. 149 (1987) 1; A. Y. Umnikov, L. P. Kaptari, K. Y. Kazakov and F. C. Khanna, arXiv:hep-ph/9410241.

30. A. Airapetian et al. [ HERMES Collaboration ], Phys. Rev. D71 (2005) 012003, [hep-ex/0407032].

31. D. de Florian, R. Sassot, M. Stratmann and W. Vogelsang, Phys. Rev. Lett. 101 (2008) 072001 [arXiv:0804.0422 [hep-ph]]; Phys. Rev. D 80 (2009) 034030 [arXiv:0904.3821 [hep-ph]]; C. Bourrely, F. Buccella, J. Soffer, [arXiv:1008.5322 [hep-ph]] and references therein; J. Soffer, these proceedings.

32. M. Glück, E. Reya, M. Stratmann and W. Vogelsang, Phys. Rev. D 63 (2001) 094005 [arXiv:hep$\mathrm{ph} / 0011215]$.

33. M. Hirai, S. Kumano and N. Saito, Phys. Rev. D 74 (2006) 014015 [arXiv:hep-ph/0603213].

34. E. Leader, A. V. Sidorov, D. B. Stamenov, Phys. Rev. D75 (2007) 074027. [hep-ph/0612360].

35. A. D. Martin, W. J. Stirling, R. S. Thorne and G. Watt, Eur. Phys. J. C 63 (2009) 189 [arXiv:0901.0002 [hep-ph]].

36. V. Ravindran, J. Smith, W. L. van Neerven, Nucl. Phys. B682 (2004) 421-456. [hep-ph/0311304]; Nucl. Phys. B704 (2005) 332-348. [hep-ph/0408315];

J. Blümlein and V. Ravindran, Nucl. Phys. B 716 (2005) 128 [arXiv:hep-ph/0501178].

37. S. Alekhin, J. Blümlein, P. Jimenez-Delgado, S. Moch and E. Reya, arXiv:1011.6259 [hep-ph].

38. S. Bethke, Eur. Phys. J. C 64 (2009) 689 [arXiv:0908.1135 [hep-ph]].

39. J. Blümlein, H. Böttcher and A. Guffanti, Nucl. Phys. B 774 (2007) 182 [arXiv:hep-ph/0607200]; Nucl. Phys. Proc. Suppl. 135 (2004) 152 [arXiv:hep-ph/0407089].

40. M. Glück, E. Reya and C. Schuck, Nucl. Phys. B 754 (2006) 178 [arXiv:hep-ph/0604116]; P. Jimenez-Delgado and E. Reya, Phys. Rev. D 79 (2009) 074023 [arXiv:0810.4274 [hep-ph]].

41. S. Alekhin, J. Blümlein, S. Klein and S. Moch, Phys. Rev. D 81 (2010) 014032 [arXiv:0908.2766 [hep-ph]].

42. S.I. Alekhin, J. Blümlein, and S.-O. Moch, PoS DIS2010 (2010) 021 [arXiv:1007.3657 [hep-ph]] and DESY 10-065, in preparation.

43. J. Blümlein and H. Böttcher, Phys. Lett. B 662 (2008) 336 [arXiv:0802.0408 [hep-ph]].

44. S.I. Alekhin, S. Kulagin and R. Petti, The low-Q deep-inelastic scattering data in the global fit of PDFs, Proc. 15th International Workshop on Deep-Inelastic Scattering and Related Subjects (DIS2007), Munich, Germany, 2007, Vol. 1, pp. 313. 